\title{
SÍNTESE E CARACTERIZAÇÃO ESTRUTURAL DE COMPLEXOS MONO E BIMETÁLICOS DE PALÁDIO E PLATINA COM LIGANTES CONTENDO NITROGÊNIO E ENXOFRE
}

\author{
Vagner Fernandes Knupp, Isa Carla Nicesio, Fernanda de Magalhães Queiroz, Robson Mendes Matos \\ e Bernadette de F. Trigo Passos \\ Departamento de Química - ICEx - Universidade Federal de Minas Gerais - 31270-901 - Belo Horizonte - MG
}

Recebido em 20/9/96; aceito em 23/1/97

\begin{abstract}
SYNTHESIS AND STRUCTURAL CHARACTERIZATION OF PALLADIUM AND PLATINUM MONO- AND BIMETALLIC COMPLEXES WITH LIGANDS CONTAINING NITROGEN AND SULFUR. The synthesis of several mono- and bimetallic platinum and palladium complexes are reported. The monometallic complexes are of the type $\left[\mathrm{MCl}_{2}\left(\mathrm{PEt}_{3}\right)_{2} \mathrm{~L}\right](\mathrm{L}=2$-aminothiazol; benzothiazol; 2-imidazolidithione; $\mathrm{M}=\mathrm{Pt}$; Pd), and the bimetallic complexes $\left(\left[\mathrm{M}_{2} \mathrm{Cl}_{4}\left(\mathrm{PEt}_{3}\right)_{2} \mathrm{~L}\right]\right)$ are derived from them. Infrared spectroscopic characterization of the monometallic complexes shows that the metal centre are coordinated through the $\mathrm{N}$-endocyclic $(\mathrm{L}=\mathbf{2}$-aminothiazol; benzothiazol) and through the sulphur when $\mathrm{L}=2$-imidazolidithione. Coordination of a second metal fragment occurs via the sulphur atom $\left(\mathrm{L}=2\right.$-aminothiazol; benzothiazol) according to infrared studies. ${ }^{31} \mathbf{P}\left\{{ }^{1} \mathrm{H}\right\}$ NMR spectroscopy suggest that the complexes have a cis-configuration since the ${ }^{1} J_{P t-P}$ are around the value found for this type of compound.
\end{abstract}

Keywords: 2-imidazolidithione; 2-aminothiazol; benzothiazol.

\section{INTRODUÇÃO}

A química de compostos organofosforados, principalmente aquela envolvendo fósforo com baixo número de coordenação, tem despertado um interesse grande nos últimos anos. O número de novos catalisadores contendo ligantes fosforados cresce a cada dia. Um grande avanço também tem sido observado no uso da espectroscopia de RMN multinuclear $\left({ }^{31} \mathrm{P} ;{ }^{195} \mathrm{Pt} ;{ }^{13} \mathrm{C}\right.$ e $\left.{ }^{1} \mathrm{H}\right)$ na caracterização de compostos organometálicos, sendo que a espectroscopia de $\operatorname{RMN}{ }^{31} \mathrm{P}\left\{{ }^{1} \mathrm{H}\right\}$ vem ocupando lugar de destaque ultimamente, por produzir espectros com facilidade e fornecer dados importantes quanto a geometria dos complexos.

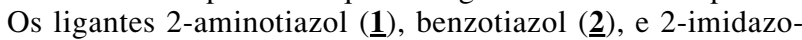
lidinationa ( $\underline{\mathbf{3}})$ são ligantes importantes na química de coordenação pois apresentam três, dois e três sítios básicos, respectivamente. Pouco ou quase nada é encontrado sobre suas reatividades frente a organometálicos de platina e paládio.<smiles>Nc1nccs1</smiles>

(1)<smiles>c1ccc2scnc2c1</smiles>

$(\underline{2})$<smiles>S=C1NCCN1</smiles>

(3)
Desta forma, objetivou-se a obtenção de compostos de coordenação de platina e paládio contendo os ligantes citados. Após a síntese e caracterização dos compostos monometálicos do tipo $\left[\mathrm{MCl}_{2}\left(\mathrm{PEt}_{3}\right) \mathrm{L}\right]$ ( $\mathrm{L}=2$-aminotiazol; benzotiazol ou 2imidazolidinationa e $\mathrm{M}=\mathrm{Pt}(\mathrm{II})$ ou $\mathrm{Pd}(\mathrm{II})$ ), decidiu-se utilizar tais complexos como ligantes na reação com os dímeros de

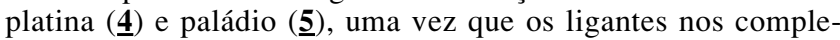
xos monometálicos ainda apresentam um ou dois sítios livres de coordenação, para a obtenção de compostos bimetálicos, os quais foram sintetizados e caracterizados, como será mostrado ao longo deste trabalho.

\section{PARTE EXPERIMENTAL}

Os compostos do tipo $\left[\mathrm{M}_{2} \mathrm{Cl}_{4}\left(\mathrm{PEt}_{3}\right)_{2}\right](\mathrm{M}=\mathrm{Pt}(\underline{4})$ e/ou $\mathrm{M}=$
Pd (ㅍ)) foram preparados segundo as sínteses descritas por Dermontt, White e Whitesides ${ }^{1}$, e Hartley ${ }^{2}$.

Os ligantes foram adquiridos comercialmente, são da marca Aldrich $^{\mathrm{R}}$ e foram utilizados sem purificação prévia.

Os espectros de absorção na região do infravermelho foram obtidos no espectrômetro Perkin-Elmer 283-B em pastilhas de CsI. Os espectros de ressonância magnética nuclear de fósforo foram obtidos no aparelho Brucker AC-80 operando na frequência $32,4 \mathrm{MHz}$ e utilizando-se $\mathrm{H}_{3} \mathrm{PO}_{4}$ como referência externa. Os pontos de fusão foram determinados no aparelho Leitz Wetzlar-Dialux, modelo 247 (sem correção) e os dados de análise elementar obtidos no aparelho microanalisador Perkin-Elmer $2400 \mathrm{CHN}$. Todos estes equipamentos pertencentes ao Departamento de Química do Instituto de Ciências Exatas da UFMG.

Todas as sínteses foram realizadas utilizando-se técnicas padrão de manipulação de materiais sensíveis ao ar usando-se atmosfera de nitrogênio, tubo Schlenck e linha de vácuo. Os resultados de análise elementar estão de acordo com as estruturas propostas.

As reações realizadas seguiram os esquemas 1,2 e 3 mostrados a seguir.

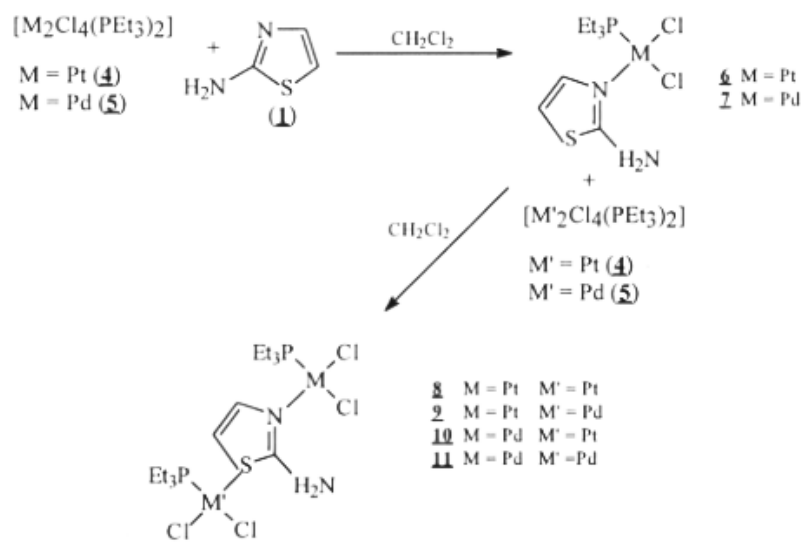

Esquema 1 


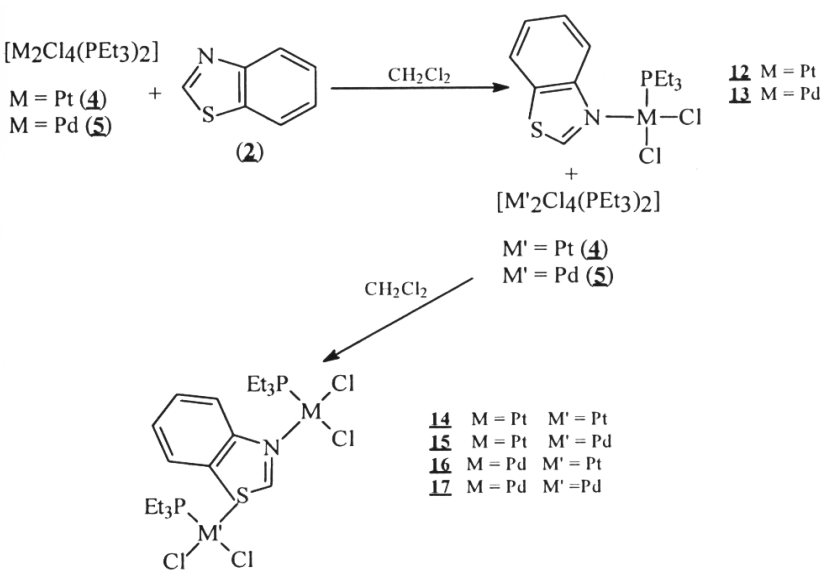

Esauema 2

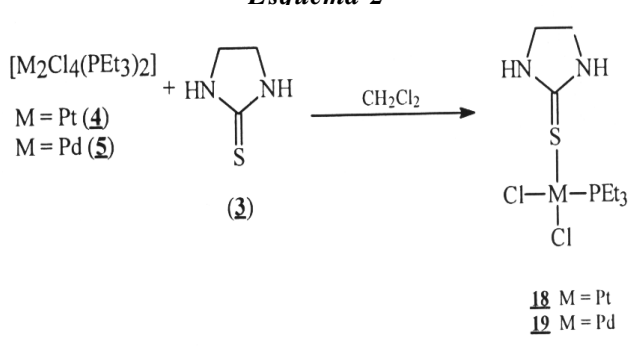

Esquema 3

\section{a. Síntese dos Compostos Monometálicos}

\section{a.1. Compostos $\underline{6}, \underline{12}$ e $\underline{18}$}

A uma solução de $0,4 \mathrm{mmol}$ do ligante $(\underline{\mathbf{1}}, \underline{\mathbf{2}}$ ou $\underline{\mathbf{3}})$ em 4,0 $\mathrm{ml}$ de $\mathrm{CH}_{2} \mathrm{Cl}_{2}$, recentemente destilado, adicionou-se uma solução de $0,2 \mathrm{mmol}$ do dímero de platina (4), em 5,0 $\mathrm{ml}$ de $\mathrm{CH}_{2} \mathrm{Cl}_{2}$. A mistura foi agitada por 24 horas. A evaporação total do solvente gerou os complexos desejados.

\section{a.2. Compostos $\underline{7}, \underline{13}$ e $\underline{19}$}

Uma solução de $0,3 \mathrm{mmol}$ do dímero de paládio (ㅁ), em $15,0 \mathrm{ml}$ de $\mathrm{CH}_{2} \mathrm{Cl}_{2}$, recentemente destilado, foi adicionada à solução de 0,6 mmol dos ligantes ( $\underline{\mathbf{1}}, \underline{\mathbf{2}}$ ou $\underline{\mathbf{3}})$, em $30,0 \mathrm{ml}$ de $\mathrm{CH}_{2} \mathrm{Cl}_{2}$. Após agitação por 24 horas evaporou-se o solvente até a secura obtendo-se um óleo, que foi lavado, em banho de ultra-som por $30 \mathrm{~min}$., com n-hexano $(3 \times 20,0 \mathrm{ml})$ resultando em um sólido cristalino.

\section{b. Síntese dos Compostos Bimetálicos}

\section{b.1. Compostos $\underline{8}, \underline{10}, \underline{14}$ e $\underline{16}$}

A uma solução de $0,3 \mathrm{mmol}$ do dímero de platina $(\underline{4}) \mathrm{em}$ $10,0 \mathrm{ml}$ de $\mathrm{CH}_{2} \mathrm{Cl}_{2}$, recentemente destilado, adicionou-se uma solução de $0,6 \mathrm{mmol}$ dos compostos $\underline{\mathbf{6}}, \underline{\mathbf{7}}, \underline{\mathbf{1 2}}$ ou $\underline{\mathbf{1 3}}$, em 10,0 $\mathrm{ml} \mathrm{de} \mathrm{CH}_{2} \mathrm{Cl}_{2}$. Após 60 minutos a solução se torna turva e a agitação é mantida por 24 horas, desaparecendo assim a turvação. O solvente é evaporado até a secura gerando um óleo o qual é lavado, em banho de ultra-som por $30 \mathrm{~min}$., com n-hexano $(3 \times 10,0 \mathrm{ml})$ para se obter o composto bimetálico como um sólido cristalino.

\section{b.2. Compostos $\underline{9}, \underline{11}, \underline{15}$ e $\underline{17}$}

A uma solução de $0,2 \mathrm{mmol}$ do dímero de paládio (5) em $10,0 \mathrm{ml}$ de $\mathrm{CH}_{2} \mathrm{Cl}_{2}$, recentemente destilado, adicionou-se uma solução de $0,4 \mathrm{mmol}$ dos compostos $\underline{\mathbf{6}}, \underline{\mathbf{7}}, \underline{\mathbf{1 2}}$ ou $\underline{\mathbf{1 3}}$, em 10,0 $\mathrm{ml}$ de $\mathrm{CH}_{2} \mathrm{Cl}_{2}$. A mistura foi agitada por 24 horas. Após a evaporação total do solvente constatou-se a presença do material de partida ( $\underline{\mathbf{5}}$ ). Assim, redissolveu-se a mistura em $20,0 \mathrm{ml}$ de $\mathrm{CH}_{2} \mathrm{Cl}_{2}$ e deixou-se em agitação por mais 24 horas. $\mathrm{O}$ solvente foi evaporado até a secura originando um óleo o qual foi lavado, em banho de ultra-som por $30 \mathrm{~min}$., com n-pentano $(3 \times 10,0 \mathrm{ml})$ para se obter o composto bimetálico como um sólido cristalino.

\section{RESULTADOS E DISCUSSÃO}

\section{a. Compostos com 2-aminotiazol (1)}

\section{a.1. Compostos monometálicos}

Os dados de espectroscopia no IV (Tabela 2) são condizentes com uma coordenação via $\mathrm{N}$-endocíclico, já que se observa uma variação no comprimento de onda da banda de estiramento $\mathrm{C}=\mathrm{N}$, em torno de $40 \mathrm{~cm}^{-1}$, em relação ao ligante livre (Figuras 1 e 2).

Uma coordenação via $\mathrm{N}$-exocíclico $\left(\mathrm{NH}_{2}\right)$ pode ser descartada já que não se detecta nenhum deslocamento da banda de

Tabela 1. Pontos de fusão, cor, análise elementar e rendimento das reações de obtenção dos compostos mono e bimetálicos.

\begin{tabular}{|c|c|c|c|c|c|c|c|c|c|}
\hline \multirow[t]{3}{*}{ Composto } & \multirow[t]{3}{*}{ P.F. $\left({ }^{\circ} \mathrm{C}\right)$} & \multirow[t]{3}{*}{ Rend. (\%) } & \multirow[t]{3}{*}{ Cor } & \multicolumn{6}{|c|}{ Análise elementar } \\
\hline & & & & \multicolumn{3}{|c|}{ Teórico $(\%)$} & \multicolumn{3}{|c|}{ Experimental $(\%)$} \\
\hline & & & & $\mathrm{C}$ & $\mathrm{H}$ & $\mathrm{N}$ & $\mathrm{C}$ & $\mathrm{H}$ & $\mathrm{N}$ \\
\hline$\underline{1}$ & $\longrightarrow$ & - & tijolo & & & & & & \\
\hline$\underline{\mathbf{2}}$ & - & & marron-esc. & & & & & & \\
\hline$\underline{\overline{6}}$ & $136-7$ & 75,0 & amarelo & 22,3 & 4,0 & 5,8 & 21,4 & 3,8 & 5,5 \\
\hline$\underline{7}$ & $125-6$ & 77,0 & amarelo & 27,3 & 4,8 & 7,0 & 27,7 & 4,7 & 6,9 \\
\hline$\underline{\mathbf{8}}$ & $192-3$ & 41,5 & amarelo & 20,8 & 4,0 & 3,2 & 19,2 & 3,7 & 3,0 \\
\hline$\overline{9}$ & 226 dec. & 61,5 & vermelho & 23,1 & 4,4 & 3,6 & 22,9 & 4,2 & 3,3 \\
\hline$\underline{\mathbf{1 0}}$ & 231-2 & 45,0 & vermelho & 23,1 & 4,4 & 3,6 & 23,0 & 4,1 & 3,3 \\
\hline$\underline{\overline{11}}$ & 236 dec. & 43,5 & vermelho & 26,1 & 5,0 & 4,1 & 25,3 & 4,8 & 3,9 \\
\hline$\underline{\overline{12}}$ & $154-5$ & 74,0 & amarelo-esv. & 28,4 & 4,0 & 2,8 & 28,0 & 3,8 & 2,5 \\
\hline$\underline{13}$ & $159-60$ & 75,0 & amarelo & 34,4 & 4,8 & 3,4 & 34,2 & 4,5 & 3,2 \\
\hline$\overline{14}$ & $149-50$ & 49,5 & amarelo-esv. & 24,3 & 4,0 & 1,6 & 23,9 & 3,8 & 1,3 \\
\hline$\underline{15}$ & $230 \mathrm{dec}$. & 68,5 & marron-av. & 26,9 & 4,4 & 1,7 & 26,6 & 4,5 & 1,5 \\
\hline$\underline{16}$ & $151-2$ & 45,0 & amarelo-esc. & 26,9 & 4,4 & 1,7 & 27,1 & 4,5 & 1,6 \\
\hline$\underline{17}$ & 235 dec. & 67,5 & laranja & 30,3 & 4,9 & 2,0 & 29,9 & 4,8 & 2,1 \\
\hline 18 & $180-2$ & 78,0 & verde-claro & 22,2 & 4,4 & 5,8 & 22,0 & 4,2 & 5,6 \\
\hline$\underline{19}$ & $156-8$ & 65,5 & amarelo & 27,2 & 5,3 & 7,0 & 27,8 & 5,4 & 7,3 \\
\hline
\end{tabular}


Tabela 2. Dados de espectroscopia de absorção na região do infravermelho $\left(\mathrm{cm}^{-1}\right)$.

\begin{tabular}{lccc}
\hline & $\underline{\mathbf{1}}$ & $\underline{\mathbf{6}}$ & $\underline{\mathbf{z}}$ \\
\hline $\mathrm{C}=\mathrm{N}$ & 1640 & 1600 & 1600 \\
$\mathrm{C}-\mathrm{S}$ & 690 & 680 & 680 \\
$\mathrm{C}-\mathrm{N}$ & 1200 & 1210 & 1210 \\
$\mathrm{M}-\mathrm{N}$ & - & 500 & 520 \\
$\mathrm{M}-\mathrm{Cl}$ & - & 360 & 340 \\
$\mathrm{~N}-\mathrm{H}$ & $3440 / 3320$ & $3420 / 3320$ & $3440 / 3320$ \\
\hline
\end{tabular}

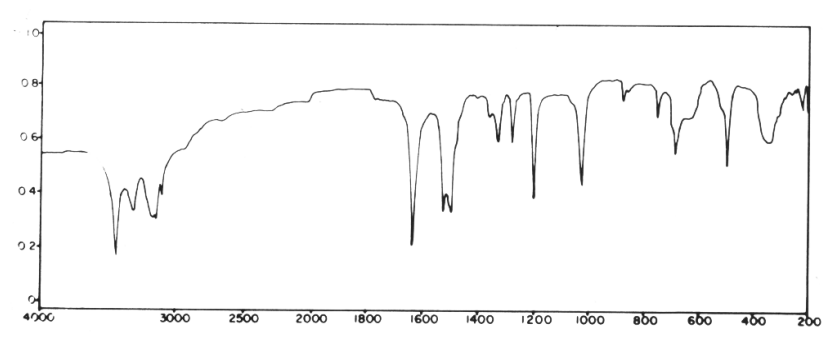

Figura 1 . Espectro de absorção na região do IV do composto (․).

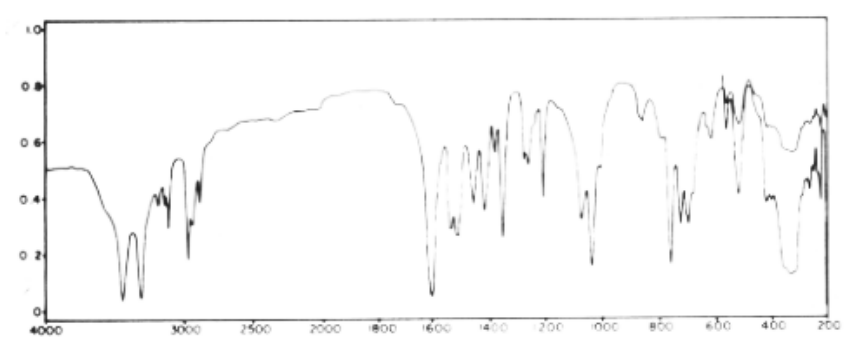

Figura 2 . Espectro de absorção na região do IV do complexo (ㅁ).

estiramento N-H no espectro de absorção na região do IV dos compostos $\underline{\mathbf{6}}$ (Figura 2) e $\underline{\mathbf{7}}$. Além disso, ocorre também um deslocamento da banda de estiramento $\mathrm{C}-\mathrm{N}$, em torno de 10 $\mathrm{cm}^{-1}$ após a complexação. Já na região de baixa frequência do espectro de absorção na região do IV desses complexos notase o aparecimento de duas novas bandas. Uma delas pode ser atribuída ao estiramento M-N $\left(500 \mathrm{~cm}^{-1}(\underline{6})\right.$ e $\left.520 \mathrm{~cm}^{-1}(\underline{7})\right)$ e

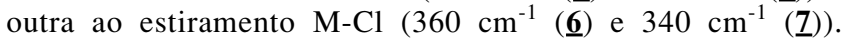
Ambas atribuições estão condizentes com os dados da literatura ${ }^{3-11}$ e confirmam a presença do fragmento $\left[\mathrm{MCl}_{2}\left(\mathrm{PEt}_{3}\right)\right]$ ao 2-aminotiazol.

Os estudos de espectroscopia de RMN ${ }^{31} \mathrm{P}\left\{{ }^{1} \mathrm{H}\right\}$ dos compostos (ㅁ) e () também sugerem a coordenação dos fragmentos $\left[\mathrm{MCl}_{2}\left(\mathrm{PEt}_{3}\right)\right]$ ao 2-aminotiazol, já que se observa uma variação no deslocamento químico do fósforo da $\mathrm{PEt}_{3}$ em relação

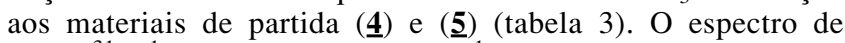
$\mathrm{RMN}{ }^{31} \mathrm{P}\left\{{ }^{1} \mathrm{H}\right\}$ de $(\underline{\mathbf{6}})$ mostra uma ${ }^{1} \mathrm{~J}_{\mathrm{Pt}-\mathrm{P}}=3436 \mathrm{~Hz}$, sugerindo uma configuração cis- ao redor da platina, já que este valor é típico para complexos que apresentam o átomo de fósforo
Tabela 3. Dados de ressonância magnética nuclear de ${ }^{31} \mathrm{P}\left\{{ }^{1} \mathrm{H}\right\}$.

\begin{tabular}{crc}
\hline Composto & $\delta_{\mathrm{P}}$ & ${ }^{1} \mathrm{~J}_{\mathrm{Pt}-\mathrm{P}}(\mathrm{Hz})$ \\
\hline$\underline{\mathbf{4}}$ & 10,4 & 3813 \\
$\underline{\mathbf{5}}$ & 9,2 & $-\overline{3436}$ \\
$\underline{\mathbf{6}}$ & $-0,53$ & - \\
\hline
\end{tabular}

trans- a cloro ${ }^{12-13}$. De um modo geral, as constantes de acoplamento Pt-P para complexos com configuração trans- são menores que aquelas apresentadas em compostos com geometria cis-. No caso de complexos com $\mathrm{Cl}^{-}$trans- a $\mathrm{PR}_{3}$, ocorre um fortalecimento da ligação Pt-P, já que os íons $\mathrm{Cl}^{-}$não são bons receptores $\pi$. Com isto é de se esperar que ${ }^{1} \mathrm{~J}_{\mathrm{Pt}-\mathrm{P}}$ para complexos com $\mathrm{P}$ trans- a $\mathrm{Cl}$ sejam maiores do que quando se tem $\mathrm{P}$ trans- a $\mathrm{P}$, já que o fósforo é um melhor receptor $\pi^{12-15}$. Por analogia, pode-se sugerir que o complexo de paládio (ㄱ) também apresente uma configuração cis-.

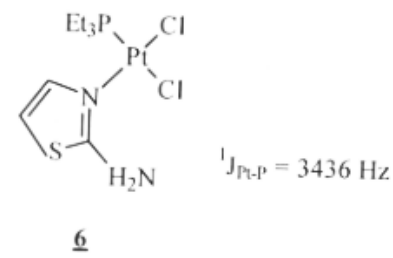

\section{a.2. Compostos bimetálicos}

Uma vez obtidas as espécies (ㅁ) e (ㅁ), as quais apresentam ainda dois outros centros de coordenação: N-exocíclico e Sendocíclico, ou seja apresentam potencial de atuarem como ligantes, resolveu-se utilizá-las na reação com os dímeros $\left[\mathrm{M}_{2} \mathrm{Cl}_{4}\left(\mathrm{PEt}_{3}\right)_{2}\right](\mathrm{M}=\mathrm{Pt}(\underline{\mathbf{4}})$ e/ou $\mathrm{M}=\mathrm{Pd}(\underline{\mathbf{5}}))$ produzindo os complexos bimetálicos $(\underline{\mathbf{8}}),(\underline{\mathbf{9}}),(\underline{\mathbf{1 0}})$ e $(\underline{\mathbf{1 1}})$. Os dados da espectroscopia de absorção na região do infravermelho (Tabela 4) condizem com as estruturas propostas, pois uma coordenação via $\mathrm{N}$-amínico pode ser descartada já que não se observa nenhum deslocamento da banda de estiramento $\mathrm{N}-\mathrm{H}^{11}$. O deslocamento da banda C-S (observado para todos os compostos) é uma indicação da ligação via S-endocíclico com os fragmentos $\left[\mathrm{MCl}_{2}\left(\mathrm{PEt}_{3}\right)\right](\mathrm{M}=\mathrm{Pt}$ ou $\mathrm{Pd})$. Além disto, observou-se também o aparecimento das bandas $\mathrm{M}-\mathrm{S}(\mathrm{M}=\mathrm{Pt}$ ou $\mathrm{Pd}$ ) e $\mathrm{M}-\mathrm{Cl}$ (quando $\left.M \neq M^{\prime}\right)^{6}$ (Figura 3).<smiles></smiles>

$\underline{\mathrm{r}} \Rightarrow \mathrm{M}=\mathrm{M}^{\cdot}=\mathrm{P}$

$2 \Rightarrow \mathrm{M}=\mathrm{Pt} \cdot \mathrm{M}=\mathrm{P}$

10 $\Rightarrow \mathrm{M}=\mathrm{M}^{\circ}=\mathrm{Pd}$

$\underline{11} \Rightarrow \mathrm{M}=\mathrm{Pd}, \mathrm{M}^{\prime}=\mathrm{Pt}$

Tabela 4. Dados de espectroscopia de absorção na região do infravermelho $\left(\mathrm{cm}^{-1}\right)$.

\begin{tabular}{|c|c|c|c|c|c|}
\hline & $\underline{1}$ & $\underline{8}$ & $\underline{9}$ & $\underline{10}$ & $\underline{11}$ \\
\hline $\mathrm{C}=\mathrm{N}$ & 1640 & 1600 & 1600 & 1600 & 1600 \\
\hline $\mathrm{C}-\mathrm{S}$ & 690 & 680 & 680 & 680 & 680 \\
\hline $\mathrm{C}-\mathrm{N}$ & 1200 & 1200 & 1210 & 1250 & 1250 \\
\hline $\mathrm{M}-\mathrm{N}$ & - & 510 & 500 & 510 & 400 \\
\hline M-S & - & 360 & 355 & 390 & 385 \\
\hline $\mathrm{N}-\mathrm{H}$ & 3440 / 3320 & $3430 / 3320$ & 3440 / 3330 & 3440 / 3320 & 3445 / 3330 \\
\hline $\mathrm{M}-\mathrm{Cl}$ & - & 385 & $400(\mathrm{Pt}) / 330(\mathrm{Pd})$ & $465(\mathrm{Pt}) / 330(\mathrm{Pd})$ & 340 \\
\hline
\end{tabular}




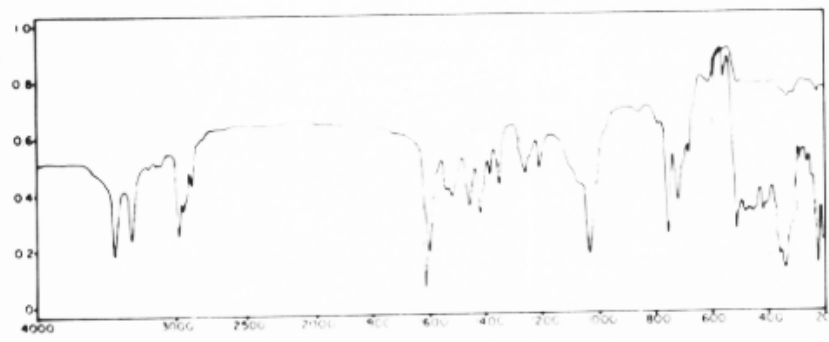

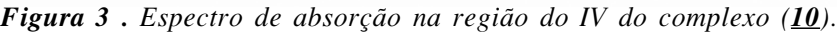

Os dados listados na tabela 5 confirmam as estruturas propostas para os compostos bimetálicos obtidos. O composto () apresenta $\delta 8,6$ para o $\mathrm{P}^{\mathrm{A}}$ e ${ }^{1} \mathrm{~J}_{\mathrm{Pt}-\mathrm{PA}}=3843 \mathrm{~Hz}$, e $\delta 7,9$ para o $\mathrm{P}^{\mathrm{B}}$ e ${ }^{1} \mathrm{~J}_{\mathrm{Pt}-\mathrm{PB}}=3840 \mathrm{~Hz}$ e os valores da constante de acoplamento Pt-P sugerem que os $\mathrm{P}^{\mathrm{A}}$ e $\mathrm{P}^{\mathrm{B}}$ estão em posição cis- em relação ao ligante 2 -aminotiazol. Já o composto $(\underline{\mathbf{9}})$ apresenta $\delta$ 8,6 para o $\mathrm{P}^{\mathrm{A}}$ e $\delta 48,3$ para o $\mathrm{P}^{\mathrm{B}} \mathrm{e}^{1} \mathrm{~J}_{\mathrm{Pt}-\mathrm{PA}}=3848 \mathrm{~Hz}$, este valor está em concordância com a estrutura sugerida para este composto. Interessante notar que após a complexação do fragmento de $[\mathrm{Pd}]$ ao S-endocíclico do complexo $\left[\mathrm{PtCl}_{2}\left(\mathrm{PEt}_{3}\right) \mathrm{L}\right](\mathrm{L}=2$ aminotiazol) observou-se uma pequena variação na ${ }^{1} J_{\mathrm{Pt} \text {-PA }} \mathrm{em}$ relação ao composto-pai (4), sugerindo um aumento no caráter s da ligação Pt-P . O composto (10 $)$ mostra um singleto a $\delta 39,1$ para o $\mathrm{P}^{\mathrm{A}}$ e um singleto com os respectivos satélites da platina a $\delta 9,2$ para o $\mathrm{P}^{\mathrm{B}}$ com constantes de acoplamento ${ }^{1} \mathrm{~J}_{\mathrm{Pt}-\mathrm{PB}}=3838$ Hz. Já o composto $(\underline{\mathbf{1 1}})$ apresenta um singleto a $\delta 36,0$ para o $\mathrm{P}^{\mathrm{A}}$ e um singleto a $\delta 48,3$ para o $\mathrm{P}^{\mathrm{B}}$. Por analogia com os compostos $(\underline{\mathbf{8}})$ e $(\underline{\mathbf{9}})$, onde ${ }^{1} \mathrm{~J}_{\mathrm{Pt}-\mathrm{P}}$ sugerem uma configuração cis-, podese inferir o mesmo para os complexos de paládio.

Tabela 5. Dados de ressonância magnética nuclear de ${ }^{31} \mathrm{P}\left\{{ }^{1} \mathrm{H}\right\}$.

\begin{tabular}{crrcc}
\hline Composto & \multicolumn{1}{c}{$\delta_{\mathrm{PA}}$} & \multicolumn{1}{c}{$\delta_{\mathrm{PB}}$} & ${ }^{1} \mathrm{~J}_{\mathrm{Pt}-\mathrm{PA}}(\mathrm{Hz})$ & ${ }^{1} \mathrm{~J}_{\mathrm{Pt}-\mathrm{PB}}(\mathrm{Hz})$ \\
\hline$\underline{\mathbf{4}}$ & 10,4 & - & 3813 & - \\
$\underline{\mathbf{5}}$ & 9,2 & - & - & - \\
$\underline{\mathbf{9}}$ & 8,6 & 7,9 & 3843 & 3840 \\
$\underline{\mathbf{1 0}}$ & 8,6 & 48,3 & 3848 & -3838 \\
$\underline{\mathbf{1 1}}$ & 36,1 & 9,2 & - & - \\
\hline
\end{tabular}

\section{b. Compostos com benzotiazol (2)}

\section{b.1. Compostos monometálicos}

Os dados de espectroscopia de absorção na região do infravermelho (Tabela 6) e espectroscopia de $\mathrm{RMN}{ }^{31} \mathrm{P}\left\{{ }^{1} \mathrm{H}\right\}$ (Tabela 7) permitem sugerir as estruturas para os compostos monometáli-

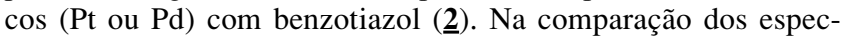
tros de absorção na região do IV do ligante livre (Figura 4) com

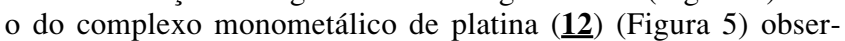
va-se o aparecimento de três novas bandas, a saber:v(Pt-N) (410 e $\left.355 \mathrm{~cm}^{-1}\right)$ e $v(\mathrm{Pt}-\mathrm{Cl})\left(325 \mathrm{~cm}^{-1}\right)$, as quais sugerem a presença do fragmento $\left[\mathrm{PtCl}_{2}\left(\mathrm{PEt}_{3}\right)\right]$ ligado ao átomo de nitrogênio do benzotiazol. Além disto, observa-se também um deslocamento da banda de estiramento $v(\mathrm{C}-\mathrm{N})$, reforçando a hipótese de ligação via o nitrogênio do ligante. Já para o complexo de paládio com o mesmo ligante (13) observa-se o aparecimento de uma banda a $340 \mathrm{~cm}^{-1}$, no seu espectro de absorção na região do IV, que pode ser atribuída ao estiramento $v(\mathrm{Pd}-\mathrm{Cl})$. Porém, não foi observado a banda referente ao estiramento vPd-N, possivelmente por esta estar sendo encoberta pela banda $v(\mathrm{Pd}-\mathrm{Cl})$. Contudo, observa-se um deslocamento na banda de estiramento $v(\mathrm{C}$ $\mathrm{N})$, sugerindo, similarmente ao complexo (12), uma coordenação do fragmento $\left[\mathrm{PdCl}_{2}\left(\mathrm{PEt}_{3}\right)\right]$ via o átomo de nitrogênio do benzotiazol. Os dados da espectroscopia de absorção na região
Tabela 6. Dados de espectroscopia de absorção na região do infravermelho $\left(\mathrm{cm}^{-1}\right)$.

\begin{tabular}{lccc}
\hline & $\underline{\mathbf{2}}$ & $\underline{\mathbf{1 2}}$ & $\underline{\mathbf{1 3}}$ \\
\hline $\mathrm{C}=\mathrm{N}$ & 1480 & 1480 & 1480 \\
$\mathrm{C}-\mathrm{S}$ & 690 & 695 & 695 \\
$\mathrm{C}-\mathrm{N}$ & $1320 / 1290$ & $1300 / 1270$ & $1300 / 1270$ \\
$\mathrm{M}-\mathrm{N}$ & - & $410 / 355$ & $\underline{240}$ \\
$\mathrm{M}-\mathrm{Cl}$ & - & 325 & 240 \\
\hline
\end{tabular}

Tabela 7. Dados de ressonância magnética nuclear de ${ }^{31} \mathrm{P}\left\{{ }^{1} \mathrm{H}\right\}$.

\begin{tabular}{ccc}
\hline Composto & \multicolumn{1}{c}{$\delta_{\mathrm{P}}$} & ${ }^{1} \mathrm{~J}_{\mathrm{Pt}-\mathrm{P}}(\mathrm{Hz})$ \\
\hline$\underline{\mathbf{4}}$ & 10,4 & 3813 \\
$\underline{\mathbf{5}}$ & 9,2 & - \\
$\underline{\mathbf{1 2}}$ & 7,2 & 3521 \\
$\underline{\mathbf{3}}$ & 37,6 & - \\
\hline
\end{tabular}

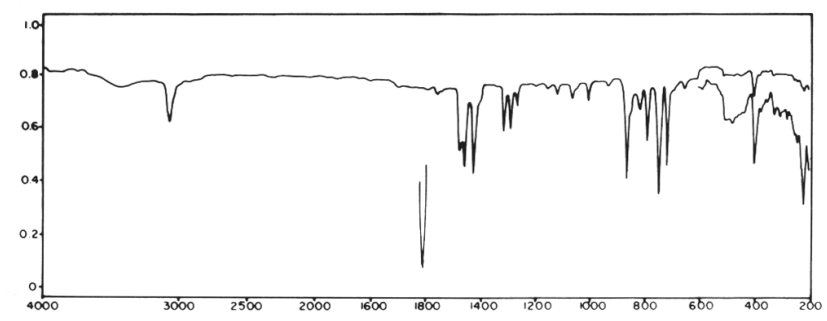

Figura 4 . Espectro de absorção na região do IV do composto (2).

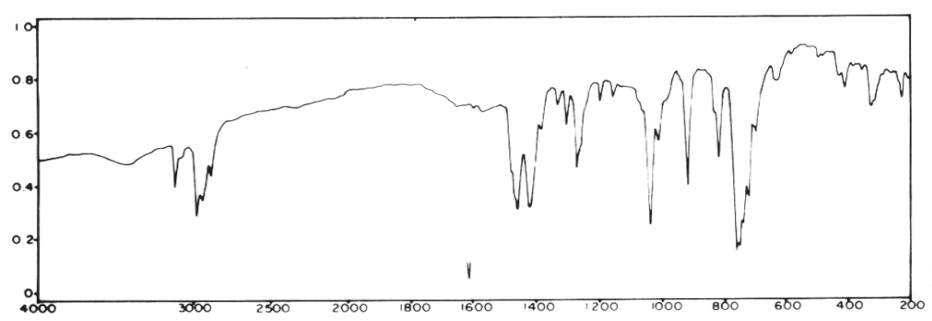

Figura 5 . Espectro de absorção na região do IV para o complexo (12).

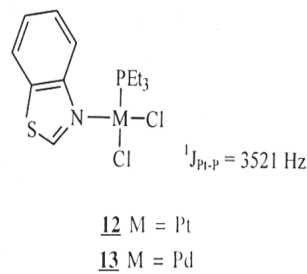

do IV para os complexos ( $\underline{\mathbf{1 2}})$ e ( $(\underline{\mathbf{1 3}})$ estão de acordo com a literatura $4,6,11,17$.

Mais evidências para a coordenação dos fragmentos $\left[\mathrm{MCl}_{2}\left(\mathrm{PEt}_{3}\right)\right](\mathrm{M}=\mathrm{Pt}$ ou Pd $)$ são obtidas a partir dos estudos de espectroscopia de RMN ${ }^{31} \mathrm{P}\left\{{ }^{1} \mathrm{H}\right\}$ (Tabela 7). Porém, mais importante que isto é a informação a respeito da configuração dos ligantes ao redor do metal, que pode ser obtida com segurança destes estudos. Para o complexo (12), observa-se um singleto a $\delta 7,2$; com os respectivos satélites da platina, fornecendo uma constante de acoplamento ${ }^{1} \mathrm{~J}_{\mathrm{Pt}-\mathrm{PA}}$ $=3521 \mathrm{~Hz}$, que como já explicado anteriormente é típica de compostos do tipo cis $^{12,15}$. Já o complexo de paládio $(\underline{\mathbf{1 3}})$ apresenta um singleto a $\delta 37,6$. Por analogia, pode-se sugerir para este composto uma configuração também do tipo cis-. 


\section{b.2. Compostos bimetálicos}

Seguindo-se a síntese dos compostos monometálicos do tipo [ $\left.\mathrm{MCl}_{2}\left(\mathrm{PEt}_{3}\right) \mathrm{L}\right](\mathrm{L}=$ benzotiazol, $\mathrm{M}=\mathrm{Pt}(\underline{\mathbf{1 2}}) \mathrm{e} / \mathrm{ou} \mathrm{M}=\mathrm{Pd}$ $(\underline{\mathbf{1 3}}))^{18}$, decidiu-se utilizar tais compostos como ligantes na reação com os dímeros de platina $(\underline{\mathbf{4}})$ e paládio $(\underline{\mathbf{5}})$ uma vez que os mesmos ainda apresentam um sítio livre de coordenação, ou seja, o S-endocíclico.

Dados da espectroscopia de absorção na região do infravermelho, tabela 8 , sugerem uma coordenação via o átomo de enxofre, por um segundo fragmento metálico. Tal afirmação baseia-se no fato de observar-se um deslocamento considerável $\left(\sim 50 \mathrm{~cm}^{-1}\right)$ na banda de estiramento $\mathrm{v}(\mathrm{C}-\mathrm{S})$ no espectro de absorção na região do infravermelho para os compostos (14)

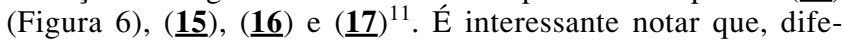
rentemente dos complexos $(\underline{\mathbf{8}})-(\underline{\mathbf{1 1}})$, naqueles de números $(\underline{\mathbf{1 4}})$ (17) observa-se uma variação na banda de estiramento $v(\mathrm{C}-\mathrm{S})$ para número de onda maiores, indicando uma maior basicidade do enxofre do ligante 2-aminotiazol comparado com o do benzotiazol. De fato isto poderia ser esperado já que o par de elétrons livres do $\mathrm{S}$ no benzotiazol está envolvido numa conjugação adicional com o anel benzênico.

Observa-se também, no espectro de absorção na região do infravermelho para o composto (14), o aumento de intensida$\mathrm{de}^{20,21,22} \mathrm{da}$ banda de estiramento $\mathrm{v}(\mathrm{Pt}-\mathrm{Cl})\left(330 \mathrm{~cm}^{-1}\right) . \mathrm{O}$ mesmo fato é observado com a banda de estiramento $\mathrm{v}(\mathrm{Pd}-\mathrm{Cl})$ para o composto $(\underline{\mathbf{1 7}})$.

Para os compostos $(\underline{\mathbf{1 5}})$ e ( $\underline{\mathbf{1 6}})$ foi detectado uma banda referente ao estiramento $v(\overrightarrow{\mathrm{Pd}}-\mathrm{Cl})\left(340 \mathrm{~cm}^{-1}\right)$ e $v(\mathrm{Pt}-\mathrm{Cl})\left(330 \mathrm{~cm}^{-1}\right)$, respectivamente, em seus espectros de absorção na região do infravermelho destes complexos. Nota-se, também, da análise da tabela 8 o aparecimento de uma nova banda de estiramento $v(\mathrm{M}-\mathrm{S})$ na região entre $378-350 \mathrm{~cm}^{-1}$ para todos os compostos bimetálicos ${ }^{6}$, confirmando, assim, a presença de mais um fragmento metálico ligado ao benzotiazol ${ }^{18}$.

Os dados apresentados na tabela 9 estão em concordância

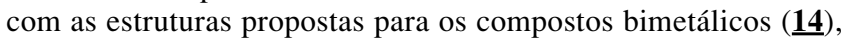
$(\underline{\mathbf{1 5}}),(\underline{\mathbf{1 6}})$ e $(\underline{\mathbf{1 7}})$.

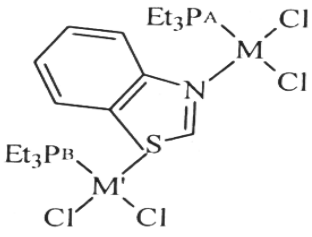

Os deslocamentos químicos $\delta 7,4\left(\mathrm{P}^{\mathrm{A}}\right), \delta 7,1\left(\mathrm{P}^{\mathrm{B}}\right)(\underline{\mathbf{1 4}}) ; \delta 7,4$ $\left(\mathrm{P}^{\mathrm{A}}\right), \delta 40,3\left(\mathrm{P}^{\mathrm{B}}\right)(\underline{\mathbf{1 5}}) ; \delta 42,0\left(\mathrm{P}^{\mathrm{A}}\right), \delta 8,4\left(\mathrm{P}^{\mathrm{B}}\right)(\underline{\mathbf{1 6}}) ; \delta \underline{41,0}\left(\mathrm{P}^{\mathrm{A}}\right)$, $\delta 43,0\left(\mathrm{P}^{\mathrm{B}}\right)(\underline{\mathbf{1 7}})$ e as constantes de acoplamento ${ }^{1} \mathrm{~J}_{\mathrm{Pt}-\mathrm{PA}}=3515$ $\mathrm{Hz},{ }^{1} \mathrm{~J}_{\mathrm{Pt}-\mathrm{PB}}=3510 \mathrm{~Hz}(\underline{\mathbf{1 4}}) ;{ }^{1} \mathrm{~J}_{\mathrm{Pt}-\mathrm{PA}}=3520 \mathrm{~Hz}(\underline{\mathbf{1 5}}) ;{ }^{1} \mathrm{~J}_{\mathrm{Pt}-\mathrm{PB}}=$ $3500 \mathrm{~Hz}(\underline{\mathbf{1 6}})$, sugerem por analogia com os compostos $(\underline{\mathbf{8}}),(\underline{\mathbf{9}})$, (10) e (11) uma configuração cis- para os bimetálicos estudados.

\section{c. Compostos com 2-imidazolidinationa ( $\underline{3})$}

\section{c.1. Compostos monometálicos}

O ligante 2-imidazolidinationa ( $\underline{\mathbf{3}})$ é uma espécie que apresenta três sítios básicos de coordenação. Na análise do seu espectro de

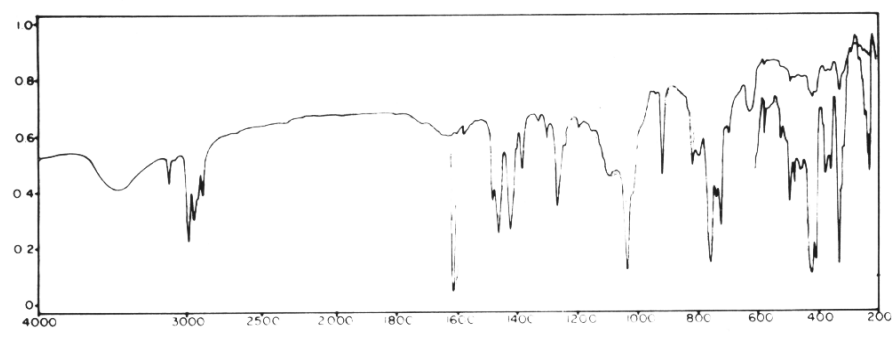

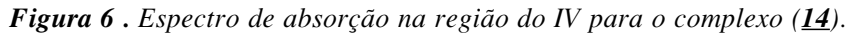

Tabela 9. Dados de ressonância magnética nuclear de ${ }^{31} \mathrm{P}\left\{{ }^{1} \mathrm{H}\right\}$.

\begin{tabular}{crrcc}
\hline Composto & \multicolumn{1}{c}{$\delta_{\mathrm{PA}}$} & \multicolumn{1}{c}{$\delta_{\mathrm{PB}}$} & ${ }^{1} \mathrm{~J}_{\mathrm{Pt}-\mathrm{PA}}(\mathrm{Hz})$ & ${ }^{1} \mathrm{~J}_{\mathrm{Pt}-\mathrm{PB}}(\mathrm{Hz})$ \\
\hline$\underline{\mathbf{4}}$ & 10,4 & - & 3813 & - \\
$\underline{\mathbf{1 4}}$ & 9,2 & - & - & - \\
$\underline{\mathbf{1 5}}$ & 7,4 & 7,1 & 3515 & 3510 \\
$\underline{\mathbf{1 6}}$ & 7,4 & 40,3 & 3520 & - \\
$\underline{\mathbf{1 7}}$ & 41,0 & 43,0 & - & - \\
\hline
\end{tabular}

absorção na região do infravermelho (Figura 7) foi detectado a presença de duas formas tautoméricas ${ }^{10}\left(\mathrm{v}(\mathrm{S}-\mathrm{H})=2575 \mathrm{~cm}^{-1}\right)$.<smiles>S=C1NCCN1</smiles>

()

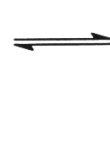<smiles>SC1=NCCN1</smiles>

( $\left.\mathbf{3}^{\prime}\right)$
Com a possibilidade de se estudar a reatividade deste ligante frente a metais de transição, optou-se pelo uso dos compostos bimetálicos do tipo $\left[\mathrm{M}_{2} \mathrm{Cl}_{4}\left(\mathrm{PEt}_{3}\right)_{2}\right]$ onde $\mathrm{M}=\mathrm{Pt}(\underline{\mathbf{4}})$ e $\mathrm{M}=\mathrm{Pd}$

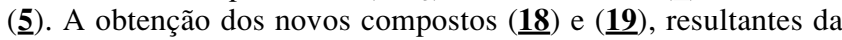
reação descrita anteriormente pode ser confirmada pela análise dos dados das tabelas 10 e 11 . Tanto para o composto ( $\underline{\mathbf{1 8}})$, quanto para o (19) (Tabela 10) ocorre o desaparecimento das bandas de estiramento $v(\mathrm{~S}-\mathrm{H})\left(2575 \mathrm{~cm}^{-1}\right)$ e $v(\mathrm{C}-\mathrm{S})\left(655 \mathrm{~cm}^{-1}\right)$ presentes no espectro de absorção na região do infravermelho do ligante (Figura 7), o que indica que a forma tautomérica (픈 é mais estável quando complexada a metais de transição ${ }^{26}$ do que a forma tautomérica ( $\left.\underline{\mathbf{3}}^{\prime}\right)$. Observando ainda a tabela 10 pode-se verificar o aparecimento das bandas de estiramento $v(\mathrm{Pt}-$ S) $\left(370 \mathrm{~cm}^{-1}\right)$ e $v(\mathrm{Pt}-\mathrm{Cl})\left(360 \mathrm{~cm}^{-1}\right)(\underline{\mathbf{1 8}}) ; \mathrm{v}(\mathrm{Pd}-\mathrm{S})\left(380 \mathrm{~cm}^{-1}\right)$ e $v(\mathrm{Pd}-\mathrm{Cl})\left(370 \mathrm{~cm}^{-1}\right)(\underline{\mathbf{1 9}})$. Nos estudos dos espectros de absorção no infravermelho dos compostos $(\underline{\mathbf{1 8}})$ e $(\underline{\mathbf{1 9}})$ (Figura 8) observase um aumento na intensidade da banda de estiramento $v(C=S)$ $\left(1040 \mathrm{~cm}^{-1}(\underline{\mathbf{1 8}}) \text { e } 1035 \mathrm{~cm}^{-1}(\underline{\mathbf{1 9}})\right)^{23,24,25}$, indicando uma complexação via $\mathrm{S}$-exocíclico do ligante.

Os dados de ressonância magnética nuclear de ${ }^{31} \mathrm{P}\left\{{ }^{1} \mathrm{H}\right\}$ para os compostos $(\underline{\mathbf{1 8}})$ e $(\underline{\mathbf{1 9}})$ se encontram listados na tabela 11. Esses resultados $\left(\delta 7,4\right.$ e ${ }^{1} \mathrm{~J}_{\mathrm{Pt}-\mathrm{P}}=3556 \mathrm{~Hz}(\underline{\mathbf{1 8}})$ e $\left.\delta 35,0(\underline{\mathbf{1 9}})\right)$ confirmam as estruturas propostas, e pelo valor de ${ }^{1} \mathrm{~J}_{\mathrm{Pt} \text {-P }}$ pode-se sugerir que o átomo de fósforo da trietilfosfina, que está ligado a platina se

Tabela 8. Dados de espectroscopia de absorção na região do infravermelho $\left(\mathrm{cm}^{-1}\right)$.

\begin{tabular}{|c|c|c|c|c|c|}
\hline & $\underline{\mathbf{2}}$ & $\underline{14}$ & $\underline{15}$ & $\underline{16}$ & $\underline{17}$ \\
\hline $\mathrm{C}=\mathrm{N}$ & 1480 & 1480 & 1485 & 1485 & 1480 \\
\hline $\mathrm{C}-\mathrm{S}$ & 690 & 740 & 740 & 740 & 740 \\
\hline $\mathrm{M}-\mathrm{N}$ & - & 410 & 410 & 400 & 400 \\
\hline $\mathrm{M}-\mathrm{S}$ & - & 378 & 375 & 355 & 350 \\
\hline $\mathrm{M}-\mathrm{Cl}$ & - & 330 & $340(\mathrm{Pt}) / 330(\mathrm{Pd})$ & $340(\mathrm{Pt}) / 330(\mathrm{Pd})$ & 340 \\
\hline
\end{tabular}


Tabela 10. Dados de espectroscopia de absorção na região do infravermelho $\left(\mathrm{cm}^{-1}\right)$.

\begin{tabular}{lccc}
\hline & $\underline{\mathbf{3}}$ & $\underline{\mathbf{1 8}}$ & $\underline{\mathbf{1 9}}$ \\
\hline $\mathrm{C}=\mathrm{S}$ & 1040 & 1040 & 1035 \\
$\mathrm{C}-\mathrm{S}$ & 655 & SOME & SOME \\
$\mathrm{M}-\mathrm{S}$ & & 370 & 380 \\
$\mathrm{~S}-\mathrm{H}$ & 2575 & SOME & SOME \\
$\mathrm{M}-\mathrm{Cl}$ & $\underline{27}$ & 360 & 370 \\
$\mathrm{C}-\mathrm{N}$ & 1275 & 1270 & 1270 \\
\hline
\end{tabular}

Tabela 11. Dados de ressonância magnética nuclear de ${ }^{31} \mathrm{P}\left\{{ }^{1} \mathrm{H}\right\}$.

\begin{tabular}{ccc}
\hline Composto & $\delta_{\mathrm{P}}$ & ${ }^{1} \mathrm{~J}_{\mathrm{Pt}-\mathrm{P}}(\mathrm{Hz})$ \\
\hline$\underline{\mathbf{4}}$ & 10,4 & 3813 \\
$\underline{\mathbf{5}}$ & 9,2 & $\overline{3556}$ \\
$\underline{\mathbf{1 9}}$ & 7,4 & - \\
\hline
\end{tabular}

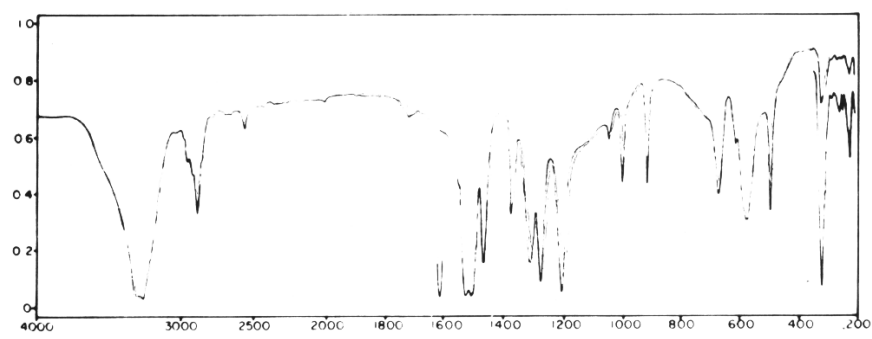

Figura 7. Espectro de absorção na região do IV do composto (3).

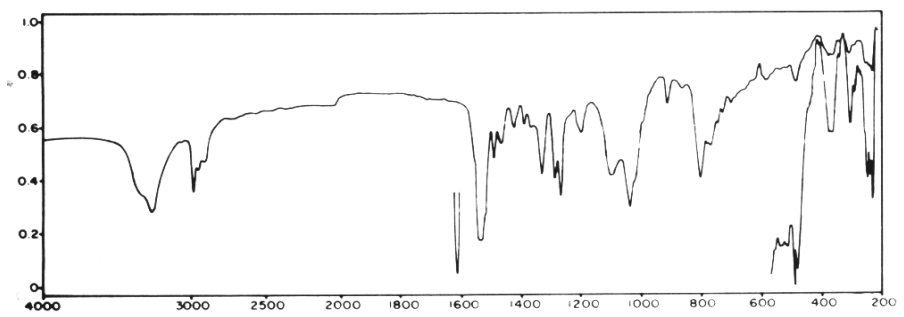

Figura 8. Espectro de absorção na região do IV do complexo (19).

encontra na posição cis- ao ligante. Por analogia, pode-se inferir o mesmo para o composto de paládio $(\underline{\mathbf{1 9}})^{12,13,14,15}$.

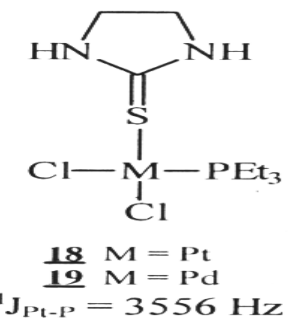

\section{AGRADECIMENTOS}

Agradecemos ao Conselho Nacional de Desenvolvimento Científico e Tecnológico (CNPq), à Financiadora de Estudos e Projetos (FINEP), à Fundação de Amparo a Pesquisa do Estado de Minas Gerais (FAPEMIG) e à Pró-reitoria de Pesquisa da Universidade Federal de Minas Gerais (PrPq-UFMG) pelo suporte financeiro recebido para a realização deste trabalho.

\section{REFERÊNCIAS}

1. Mc. Dermont, J. X.; White, J. F.; Whitesides, G. M.; J. Am. Chem. Soc. 1976, 98, 6521.

2. Hartley, F. R.; Organomet. Chem. Rev. A 1970, 6, 119.

3. Trigo Passos, B. F.; Síntese e caracterização de compostos organoestânicos envolvendo fosfinas mono-, bi-, e tridentadas. Tese de doutorado, Departamento de Química-ICEx-UFMG, Belo Horizonte MG, 1989.

4. Matos, R. M.; Estudos da complexação de algumas tioalcanaminas com metais de transição. Dissertação de mestrado, Departamento de Química-ICEx-UFMG, Belo Horizonte MG, 1989.

5. Matos, R. M.; Filgueiras, C. A. L.; Trans. Met. Chem. 1990, 15,297

6. Nakamoto, K.; Infrared and Raman Spectra; John Wiley e Sons; New York, 1986; $4^{\text {nd }}$ edition.

7. Mijovic, M. P. V.; Walker, J.; J. Chem. Soc. 1961, 3381.

8. Angyal, C. L.; Werner, R. L.; J. Chem. Soc. 1952, 2911.

9. Davidovics, G.; Choutean, J.; Spectroch. Acta 1966, 22, 703.

10. Choutean, J.; Davidovics, G.; Metzger, J.; Bonzon, A. Spectroch. Acta 1966, 22, 719.

11. Silverstein, R. M.; Bassler, G. C.; Morrill, T. C.; Spectrometric Identification of Organic Compounds; John Wiley e Sons; New York, 1981; $4^{\text {nd }}$ edition.

12. Nixon, J. F.; Pidcock, A.; Annual Review of NMR Spectroscopy 1969, 2, 459.

13. Matos, R. M.; Novel compounds derived from phosphaalkynes. Tese de doutorado, School of Chemistry and Molecular Sciences, University of Sussex, Falmer Brighton, England, 1993.

14. Verkade, J. G.; Quin, L. D.; Methods in Stereochemical Analysis; VCH; Deerfield Beach, Florida, 1987.

15. Ajulu, F. A.; Bartsch, R.; Carmichael, D.;Johnson, J. A.; Jones, C.; Matos, R. M.; Nixon, J. F.; In Phosphorus-31 NMR Spectral Properties in Compound Characterization and Structural Analysis; Verkade, J. G.; Quin, L. D., Ed; VCH; New York, 1994; p 229.

16. Knupp, V. F.; Trigo Passos, B. F.; Matos, R. M.; $9^{\circ}$ Encontro Regional da Sociedade Brasileira de Química - MG - livro de resumos, p 98, 1995.

17. Metzger, J; Cherrier, C.; Compt. Rend. 1949, $228,239$.

18. Nicesio, I. C.; Oliveira, A. M.; Matos, R. M.; Trigo Passos, B. F.; Filgueiras, C. A. L.; $17^{\text {a }}$ Reunião Anual da Sociedade Brasileira de Química - VII Simpósio Nacional de Química Inorgânica - livro de resumos, QI 190, 1994.

19. Nicesio, I. C.; Matos, R. M.; Trigo Passos, B. F.; $18^{\text {a }}$ Reunião Anual da Sociedade Brasileira de Química - $7^{\circ}$ Encontro Brasileiro de Fotoquímica e Fotobiologia - livro de resumos, QI - 45, 1995.

20. Mc Dermontt, J. X.; White, J. F.; Whitesides, G. H.; J. Am. Chem. Soc. 1976, 98, 6521.

21. Lawson Jr., J. K.; J. Am. Chem. Soc. 1953, 75, 3398.

22. van Tilborg, M. W. E. M.; van Houte, J. J.; van Thuijl, J.; Org. Mass Spect. 1984, 19, 16.

23. Caitos, H. A.; Org. Synth. 1948, 26, 16.

24. Mitchell, T. N.; Amamria, A.; Killing, H.; Rutschow, D.; J. Organomet. Chem. 1986, 304, 257.

25. Bruce, M. I.; Hameister, C.; Swincer, A. G.; Wallis, R. C.; Ittel, S. D.; Inorg. Synth. 1982, 21, 78.

26. Bouças, C. H. S.; Queiroz, F. M; Matos, R. M.; Trigo Passos, B. F.; $8^{\circ}$ Encontro Regional da Sociedade Brasileira de Química - MG - livro de resumos, p 70, 1994.

27. Queiroz , F. M.; Trigo Passos, B. F.; Matos, R. M.; $18^{\mathrm{a}}$ Reunião Anual da Sociedade Brasileira de Química - $7^{\circ}$ Encontro Brasileiro de Fotoquímica e Fotobiologia - livro de resumos, QI - 16, 1995. 